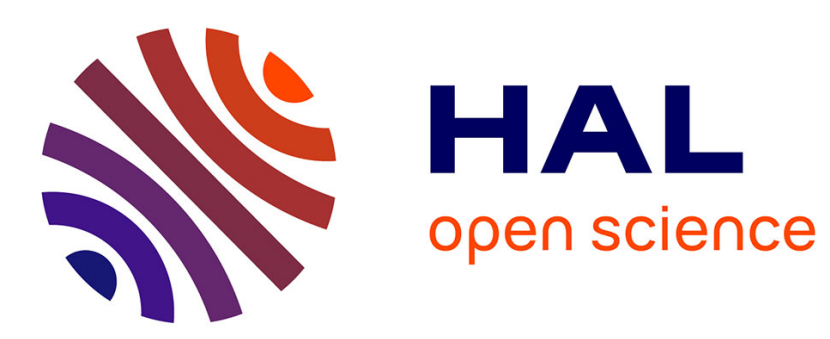

\title{
RYDBERG ATOMS INTERACTING WITH INTENSE NON RESONANT E.M. FIELD
}

\author{
J. Pinard, S. Liberman, Ahmed Taleb
}

\section{To cite this version:}

J. Pinard, S. Liberman, Ahmed Taleb. RYDBERG ATOMS INTERACTING WITH INTENSE NON RESONANT E.M. FIELD. Journal de Physique Colloques, 1982, 43 (C2), pp.C2-407-C2-412. 10.1051/jphyscol:1982231. jpa-00221843

\section{HAL Id: jpa-00221843 https://hal.science/jpa-00221843}

Submitted on 1 Jan 1982

HAL is a multi-disciplinary open access archive for the deposit and dissemination of scientific research documents, whether they are published or not. The documents may come from teaching and research institutions in France or abroad, or from public or private research centers.
L'archive ouverte pluridisciplinaire HAL, est destinée au dépôt et à la diffusion de documents scientifiques de niveau recherche, publiés ou non, émanant des établissements d'enseignement et de recherche français ou étrangers, des laboratoires publics ou privés. 


\title{
RYDBERG ATOMS INTERACTING WITH INTENSE NON RESONANT E,M, FIELD
}

\author{
J. Pinard, S. Liberman and A. Taleb \\ Laboratoire Aimé Cotton, Centre National de Za Recherche Scientifique, \\ Batiment 505, 91405 Orsay Cedex, France
}

Résumé. - Les déplacements de niveaux atomiques faiblement liés (Etats de Rydberg) dus à une perturbation par un champ électromagnétique intense, non résonnant et de faible fréquence, ont été étudiés expérimentalement. Cet effet peut être interprété par la mécanique classique de l'électron comme un accroissement de son énergie ciném tique moyenne da à ses vibrations dans le champ E.M. L La comparaison des résultats expérimentaux avec les calculs sont satisfaisants.

Abstract. - Shifts of weakly bound states of atoms (Rydberg states) due to perturbation by an intense non resonant low frequency electromagnetic field has been experimentally studied. This effect can be interpreted in terms of classical mechanics as an increase of the kinetic energy of the electron vibrating in the $E . M$. field. Comparison of experimental results with theoretical calculations has been found quite satisfactory.

Interaction of an atom with a strong electromagnetic field has been widely studied for a long time both theoretically and experimentally, especially since the discovery of laser. The main result of these interaction is the well known light shift which has given rise to numerous experiments and calculations particularly in the case where only few levels are involved in the interaction. In that case, the light shift may be calculated using the perturbation theory and the few oscillator strengths connecting the levels of interest. The corresponding experiments have been done on the deep levels of atoms which can be considered as sufficiently isolated.

However, during the past few years, the spectroscopic studies of Rydberg states has been extensively developed. For such studies it has been necessary to take into consideration the perturbation of these particular states by E.M. field. As an example, the effect of blackbody radiation mainly on the lifetime of the states has already been studied $[1,2]$. In the present work we are interested in studying perturbations due to intense non resonant laser radiation.

In any case the problem is not so simple than the above mentioned one due to the fact that the interaction involves an infinite set of levels including the continuum. In fact in a theoretical paper [3] $\mathrm{C}$. Cohen-Tannoudji and his coworkers has treated a quasi-similar problem namely the interaction of a free or weakly bound electron with high frequency E.M. field. The fundamentel hypothesis was that the extension $\Delta \mathrm{E}$ of the infinite set of levels of interest is much smaller than the energy of the perturbing photons $i \omega: \Delta E<<\pitchfork \omega$. In this particular case, using an effective hamiltonian an exact quantum treatment can be done. In such a treatment, terms corresponding to effects due to vacuum fluctuations as well as those due to an external E.M. field appear simultaneously. The first order term of the interaction is $\varepsilon_{V}=\frac{e^{2} E^{2}}{4 m \omega^{2}}$ depending on the electron charge and mass and on the amplitude of the electric field of frequency $\frac{\omega}{2 \pi}$. This term can be easily understood using classical mechanics : if one considers an 
electron moving slowly in a coulomb potential (weakly bound electron) the net effect of the high frequency E.M. field is to subject the electron to rapid forced oscillations and then the non perturbed kinetic energy is increased by an amount $\xi_{v}$ which is nothing but its vibrational kinetic energy ; it is, in fact, this term that we are mainly considering here, it is quite different from the term corresponding to the fact that the oscillating electron does not see the value of the potential at the mean position but rather a mean value of it, the corresponding correction is much lower than the previous one. However, contrary to the main term which affects equally each energy level this one depends on the potential gradient and thus on the characteristics of the electron trajectory : it will be different for an $s$ state, for which the electron probe the field at the nucleus, than for a $p$ state. This effect has a particular interpretation in the sense that the relative shift between the $s$ and $p$ state can be considered as a stimulated Lamb shift.

As mentioned above we were mainly concerned to give an experimental evidence of the increase $\boldsymbol{t}_{\mathrm{V}}$ of the electron kinetic energy. The corresponding shift of its energy level is not very small and can be easily experimentally detected using high resolution spectroscopy technics : one can calculate that with a laser at $1.06 \mu$ delivering a power density of $1 \mathrm{NW} / \mathrm{cm}^{2}$ it is of the order of $25 \mathrm{MHz}$. However the high frequency condition : $\hbar \omega \gg \Delta \mathrm{E}$ is rather difficult to achieve in atomic systems. An ideal condition would be fulfilled with atoms in a high value of the quantum number $\ell$ such as $\Delta \mathbb{E}=\frac{\mathrm{R}}{\ell^{2}} \ll \pi \omega$; but, in this case, the analysis of the perturbed state from the ground ${ }^{2}$ state would require multiphoton excitation and thus much higher laser power on a single mode at relatively low frequency. The experiment that we have attempted was done on atomic rubidium perturbed by the $1.06 \mu$ radiation of a NdYag laser. The studied Rydberg level was a $p$ one directly excited and analysed from the ground state using UV radiation. On the level scheme shown Figure (1) one can see that only few levels to not fulfil the high frequency condition and that mainly two phenomena may perturb the observation of the level shift $\xi_{V}$ through a change in the UV transition : first of all the $1.06 \mu$ radiation induces a shift of the ground state due to the resonance $5 p$ level this shift has an opposite sign compared to the shift $\mathfrak{t}_{\mathrm{V}}$. Unfortunately it cannot be measured expe-

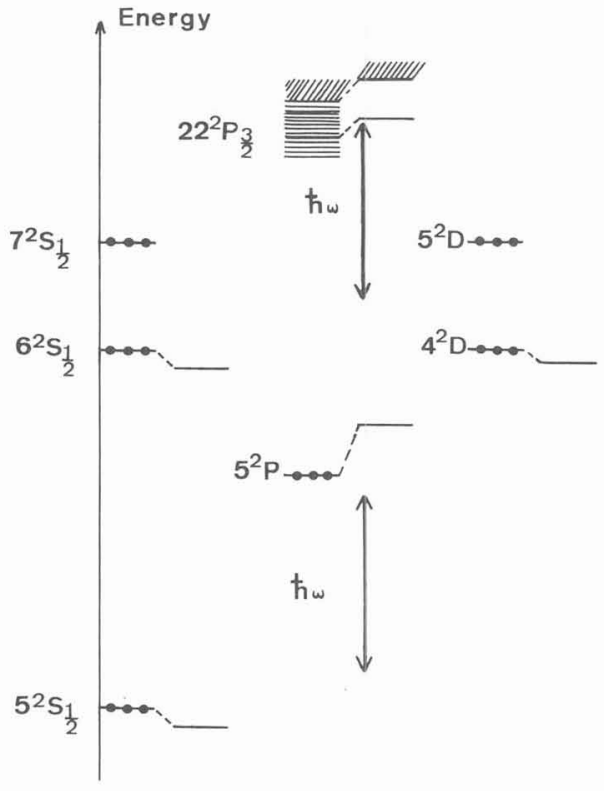
rimentally by studying the shift of the frequency of the resonance transition; the shift of the $5 p$ level being itself strongly dependent of other levels such as the $6 \mathrm{~s}$ and $4 d$. The shift of the ground state has therefore to be evaluate theoretically.

\section{Figure 1}

Shifts of the atomic energy levels of rubidium under $1.06 \mu$ excitation. 
On the other hand the shift of the Rydberg level integrates the shift we are dealing with to eventual other shifts coming from nearly resonant coupling. In fact only few valence levels may have some significant role in the presence of the $1.06 \mu$ radiation. They are : the $6 \mathrm{~s}, 7 \mathrm{~s}, 4 d$ and $5 \mathrm{~d}$, which all are quite far from resonance. Nevertheless we have to give an estimate of their influence.

The experiment has been performed on an atomic beam of rubidium atoms especially mounted for high resolution experiments [4]. As shown in Figure 2 the $\mathrm{Rb}$ atoms cross an interaction region in which they experience two laser fields : an UV one which can be tuned to the frequency of the atomic transition between the ground state $5 \mathrm{~s}$ and the Rydberg state, and an intense non resonant perturbing laser field at $1.06 \mu$. The highly excited atoms are detected by means of electric field ionization. The interaction region is actually located between capacitor plates on which a voltage pulse is applied immediately after the laser excitation. For a high enough voltage, ionization occurs and the ions are deflected towards an electron multiplier and counted.

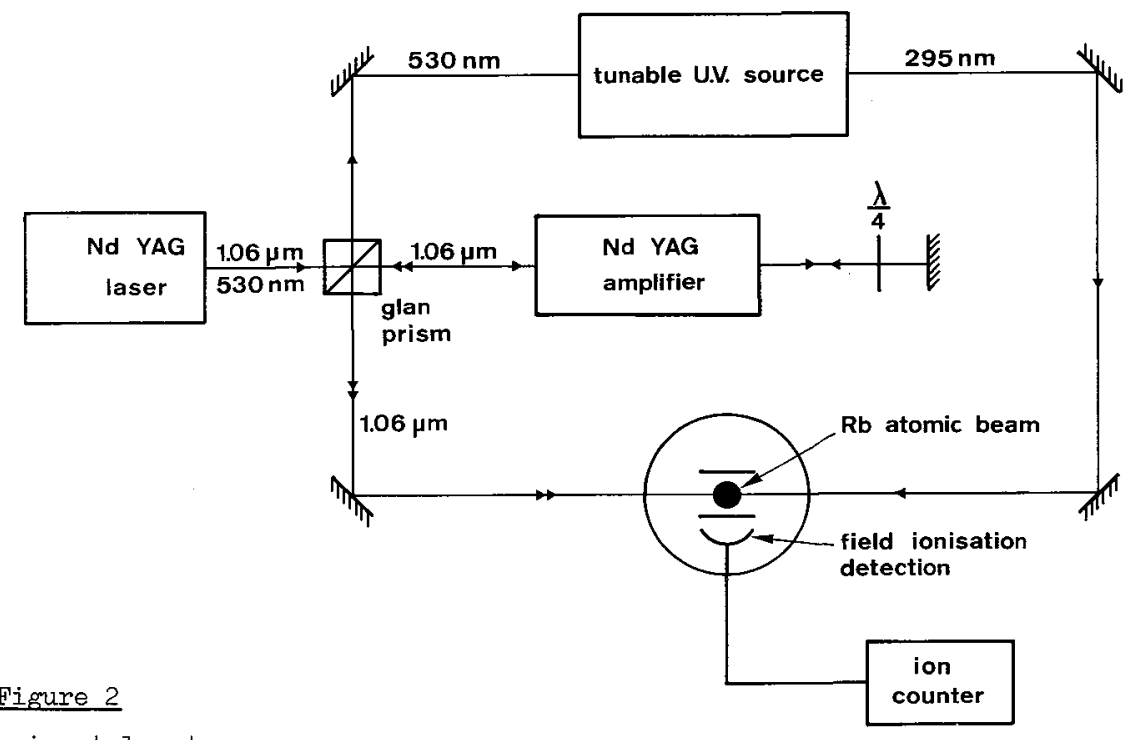

Experimental setup.

In order to overcome all synchronization difficulties the two laser fields are both generated by the same pump. The UV source at $\approx 2950 \AA$ is built from a single mode tunable pulsed dye laser using a doubling crystal (A.D.A.). It has been described in details previously [5]; it combines in some way the properties of the $\mathrm{CW}$ laser and those of a pulsed one excited by a NdYag laser doubled in frequency $(530 \mathrm{~nm})$. Its main characteristics lie in that it delivers single mode light pulses of $20 \mathrm{~ns}$ duration with a repetition rate of $10 \mathrm{~Hz}$ and a spectral linewidth of about $50 \mathrm{MHz}$ (at $295 \mu \mathrm{m}$ ). The intense IR source is provided by the residual infrared output energy at $1.06 \mu \mathrm{m}$ available at the output of the NdYag laser : the light pulse is sent into a double pass NdYag amplifier in which the use of a Glan prism together with a quater wave plate permits to extract all the energy and to decouple the amplifier from the oscillator. Such an arrangement has made it possible to deal with available IR light pulses of 140 ns duration and peak power rangring between 0 and $12 \mathrm{IVW} / \mathrm{cm}^{2}$. Both light beams (the UV one and the IR one) propagate collinearly but in opposite directions. Special care has been taken in order to make sure that the UV beam actually probed the centre of the infrared one. On the other hand a synchroneous photograph of the two pulses displayed on a scope 
screen insured us that the UV pulses was probing the highest energy part of the IR pulses. As the frequency shift measurements are depending on the power density of the IR beam and, in addition, as they have to be done in an absolute way, the previously described adjustment as well as the determination of the power density in the probed region are certainly the most delicate parts of the experiment. Actually the laser power has been measured using a power meter (Scientech) outside the atomic beam apparatus and correction has been brought to take into account the absorption of the windows. The peak power has been deduced using the pulse duration measured on the scope screen, whereas the diameter of the laser spot has been measured using a detector mounted on a precision translator. It has been found to the $.8 \mathrm{~mm}$ at half energy points.

The measurements have been done on the transition $5 s^{2} S_{1 / 2}-22 p^{2} P_{3 / 2}$ of the most abundant isotope of $\mathrm{Rb}$ (which is the $85 \mathrm{Rb}$ in the natural mixture). Each recorded spectrum shows a doublet structure which corresponds to the hyperfine splitting of the $5 \mathrm{~s}$ ground state. Its value has been measured precisely long time ago and found to be $3035 \mathrm{MHz}$; it allows us to calibrate the frequency scale in each recorded spectrum. Figure 3 shows a typical recording of the hyperfine structure of the line $5 \mathrm{~s} \leftrightarrow 22 \mathrm{p}$ in the absence $\left(P_{I R}=0\right)$ or in the presence of the infrared power. All resonances have been recorded during the same frequency scan which makes it easy to have an absolute measurement of the frequency shift. As we can see the spectral resolution is less than $60 \mathrm{MHz}$ in the UV range, which is much lower than the shift. We may observe that in the presence of the infrared power the resonances are slightly broadened : their widths are about $120 \mathrm{MHz}$ large. This broadening has been interpreted as due to both residual inhomogeneity of the probed part of the infrared beam and to fluctuations of the infrared peak power. In any case all these effects give a relative broadening with respect to the frequency shift of the order of $10 \%$.

On the Figure 4 we have plotted the result of several measurements of the frequency shift of the transition for various infrared peak power densities. As it was expected the experimental points lay on a straight line. Which passes through the origin and has a slope equal to $61 \mathrm{NHz} / \mathrm{MWcm}^{-2}$.

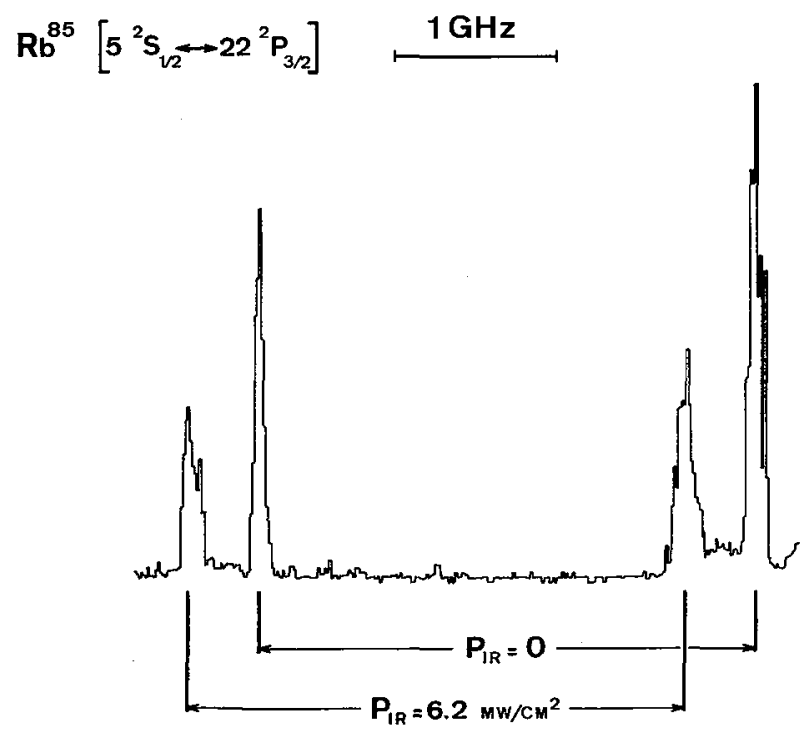

Figure 3 : Typical recording obtained by switching on and of the IR power during the same frequency scan. 
As it has been mentioned above, this frequency shift is in fact the sum of two terms, the shift of the ground state $\Delta \nu_{G}$ and the shift of the $22 \mathrm{p}$ level $\Delta \nu_{e}$ which integrates the shift we are dealing with to eventual perturbations coming from nearly resonant coupling. In order to compare the experimental results with the theory we have calculated all of these shifts. This has been done using appropriate wavefunctions which describe the energy spectrum of $\mathrm{Rb}$ atom [6]. All these data are reported in Table $I$.

\begin{tabular}{|c|c|c|}
\hline \multicolumn{2}{|c|}{ Level } & $\begin{array}{c}\text { Frequency shift } \\
\text { in MHz/MWcm }\end{array}$ \\
\hline \multirow{2}{*}{$22 p$} & $\varepsilon_{\mathrm{v}}$ & 25 \\
\cline { 2 - 3 } & $\Delta v_{5 \mathrm{~d}}$ & $310^{-3}$ \\
\hline $5 \mathrm{~s}$ & & -26.3 \\
\hline $5 p$ & & 107 \\
\hline
\end{tabular}

Table I

As we can see the contribution of the $5 \mathrm{~d}$ state to the shift of the $22 \mathrm{p}$ is completely negligible and thus a fortiori those of the $6 \mathrm{~s}, 7 \mathrm{~s}$ and $6 \mathrm{~d}$ states . On the other hand the shift of the $5 \mathrm{~s}$ ground state is of the same order of magnitude than the expected shift; we have also indicated the calculated shift of the $5 p$ state, one can remark that it is completely different from the 5 s one because it is strongly perturbed by the $5 \mathrm{~d}$ and $6 \mathrm{~s}$ states. For these reason the shift of the $5 \mathrm{~s}$ state cannot be simply measured by studying the transition $5 \mathrm{~s} \leftrightarrow 5 \mathrm{p}$.

The dotted line on Figure 4 corresponds to what one can calculate using the data of Table I. Comparison between experimental and theoretical results may be qualified as satisfactory.

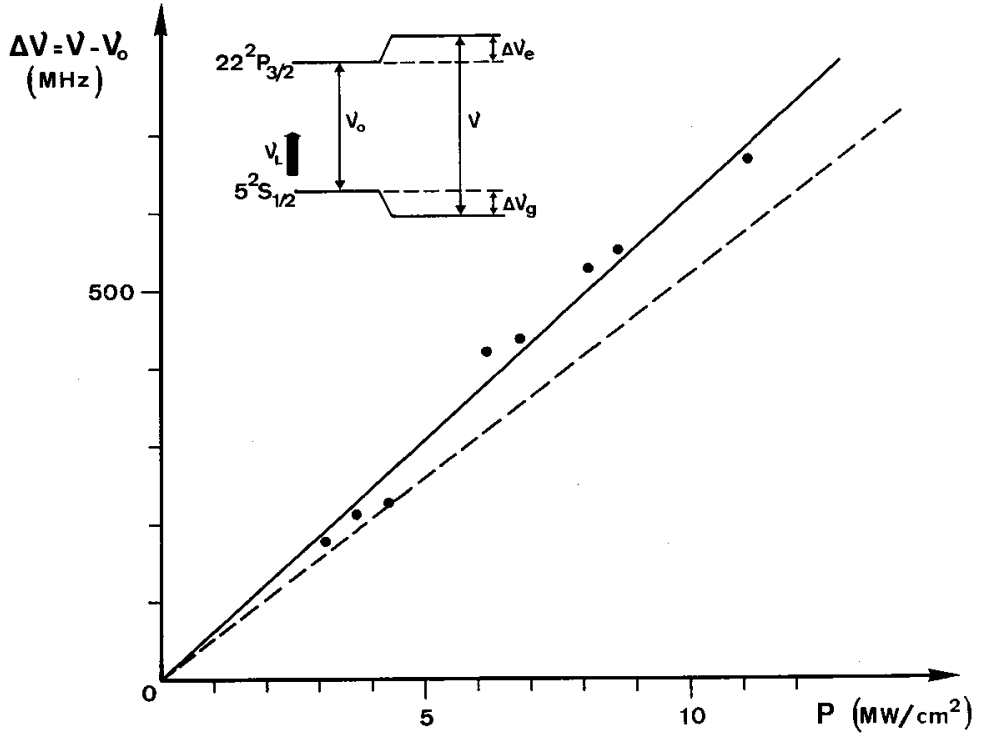

Figure 4 : Experimental measurements of the frequency shift of the UV transition. Comparison with calculated shift (dashed line). 
In conclusion we have clearly demonstrated that in the presence of a strong non resonant E.M. field, Rydberg states are shifted and that this shift may be seen as resulting from the stimulated radiative corrections induced by the field on a free or weakly bound electron.

\section{References.}

[1] T. F. Gallagher and W. E. Cooke, Phys. Rev. Lett. 42, 835 (1979).

[2] J. W. Farley, W. H. King, Phys. Rev. A 23, 2397 (1981).

[3] P. Avan, C. Cohen-Tannoudji, J. Dupont-Roc and C. Fabre, J. Physique (Paris) 37, $993(1976)$.

[4] S. Liberman and. J. Pinard, Phys. Rev. A 20, 507 (1979).

[5] S. Liberman and J. Pinard, Optics Commun. 20, 344 (1977).

[6] M. Aymar and M. Crance, private communication. 